\title{
Breakthrough curve tailing in a dipole flow field
}

\author{
Jian Luo, ${ }^{1}$ Marco Dentz, ${ }^{2}$ Olaf A. Cirpka, ${ }^{3}$ and Peter K. Kitanidis ${ }^{4}$ \\ Received 7 October 2006; revised 5 February 2007; accepted 6 June 2007; published 7 September 2007.
}

[1] Studying the tailing behavior of breakthrough curves (BTCs) is useful in the characterization of anomalous transport, matrix diffusion, and kinetic sorption. We analyze how BTCs of nonreactive and sorbing tracers behave at late time in well-to-well flow fields in homogeneous media. In the absence of regional flow, an asymptotic solution is derived for the traveltime distribution which follows a power law with exponent 4/3 at late times. With regional flow, the traveltime distribution exhibits a power law with exponent $4 / 3$ over a certain period of time, followed by exponential decay. Dispersion influences the early time behavior but has little effect on the late-time tailing. We also consider the BTC tailing of tracers undergoing kinetic sorption and diffusive mass transfer into immobile regions. If the memory function characterizing sorption kinetics is exponential, the late-time behavior of the BTC is controlled by the traveltime distribution and thus follows a power law with exponent $4 / 3$. In case of a matrix diffusion model, the memory function exhibits a power law with exponent $1 / 2$, and the BTC in the dipole flow field follows a power law with exponent $7 / 6$ in an intermediate time range, which differs from the exponent of $3 / 2$ observed in uniform flow. Our analysis

demonstrates that the flow configuration has to be considered when the tailing behavior of BTCs is used to characterize mass transfer kinetics. In particular, truncating a well-to-well tracer test at times where the traveltime distribution still follows the power law behavior may lead to the erroneous interpretation that the power law tailing of the BTC is caused by matrix diffusion.

Citation: Luo, J., M. Dentz, O. A. Cirpka, and P. K. Kitanidis (2007), Breakthrough curve tailing in a dipole flow field, Water Resour. Res., 43, W09403, doi:10.1029/2006WR005600.

\section{Introduction}

[2] The late-time tailing behavior of breakthrough curves (BTCs) has gained attention in hydrogeology. For nonreactive tracer transport, long BTC tails may result from the tracer injection mode, aquifer heterogeneities, kinetic mass transfer or diffusion between mobile and stagnant zones, among others. The mode of tracer injection significantly influences BTC tailing [Widestrand et al., 2007]. This impact can easily be identified by taking measurements at the inlet of the system. The most common injection mode consists of an injection with finite duration. While mixing in the inlet may lead to an exponentially decaying inflow concentration, its effect on the BTC may be neglected in many applications [e.g., Widestrand et al., 2007]. In homogeneous media, kinetic mass transfer between immobile water in matrix pores and mobile water in preferential flow paths is usually considered as the primary mechanism responsible for long breakthrough tails [Haggerty et al.,

\footnotetext{
${ }^{1}$ School of Civil and Environmental Engineering, Georgia Institute of Technology, Atlanta, Georgia, USA.

${ }^{2}$ Department of Geotechnical Engineering and Geosciences, Technical University of Catalonia, Barcelona, Spain.

${ }^{3}$ Swiss Federal Institute of Aquatic Science and Technology, Dübendorf, Switzerland.

${ }^{4}$ Department of Civil and Environmental Engineering, Stanford University, Stanford, California, USA.
}

Copyright 2007 by the American Geophysical Union. 0043-1397/07/2006WR005600\$09.00
2000]. After the tracer peak has passed, the retention of tracer resulting from kinetic mass transfer starts to dominate the BTC, causing a long tail of low concentrations. Thus the tailing behavior can be used to identify an appropriate model for the description of kinetic mass transfer [Haggerty et al., 2000], to characterize the retention properties caused by diffusion into the rock matrix in fractured aquifers [Hadermann and Heer, 1996; Haggerty et al., 2001], and to study sorption/desorption properties for sorbing tracers [Dunnivant et al., 1992; Farrell and Reinhard, 1994; Werth et al., 1997].

[3] In heterogeneous media, the spatially variable velocity field can cause anomalous transport, which is also characterized by extended tracer BTC tails [e.g., Selroos and Cvetkovic, 1992; Berkowitz and Scher, 1997, 1998; Berkowitz et al., 2000; Guswa and Freyberg, 2000; Di Donato et al., 2003; Dentz et al., 2004; Di Donato and Blunt, 2004; Zinn et al., 2004; Berkowitz et al., 2006; Fiori et al., 2006]. The highly asymmetric BTCs cannot be described well by the classical advection-dispersion equation (ADE) with uniform velocity and dispersion coefficient. Here, part of the tracer mass moves quickly along preferential flow paths, resulting in early first arrival and even an early concentration peak, whereas a substantial portion of the tracer mass moves slowly along low-velocity paths, causing a long BTC tail. Levy and Berkowitz [2003] showed that even tracer BTCs in homogeneous, saturated sand may be too asymmetric to be fully explained by advective-dispersive transport with uniform coefficients. 


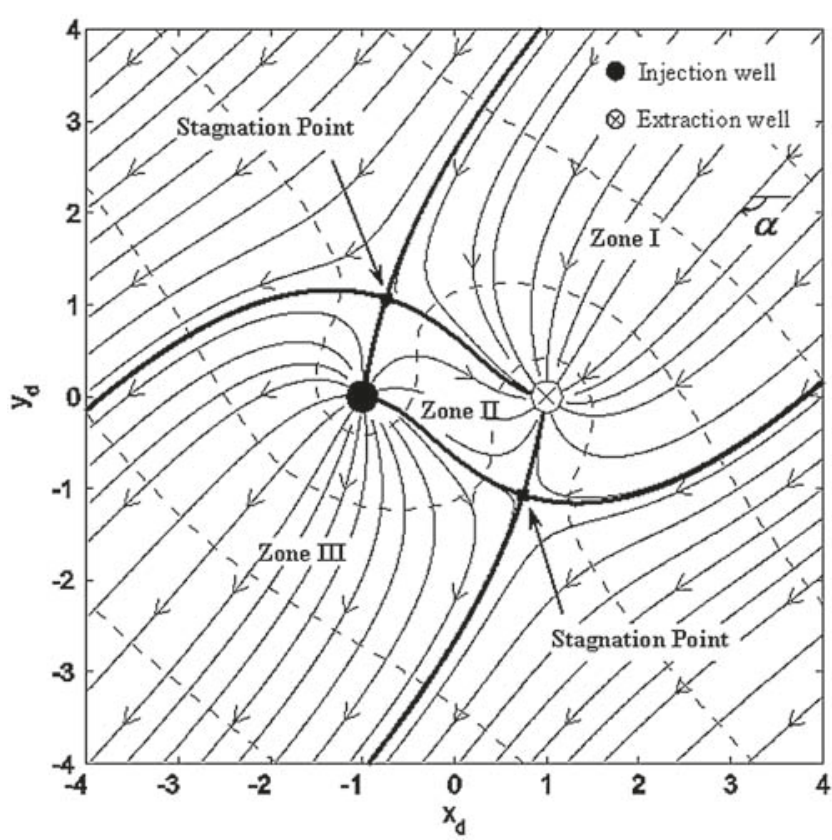

Figure 1. Plan view of the flow field created by an extraction-injection well pair with uniform regional flow in a dimensionless domain. Thin solid lines are streamlines; dashed lines are hydraulic equipotential lines; and thick solid lines are separation streamlines; $\alpha$ is the regional flow orientation. Zone I is the capture zone; zone II is the recirculation zone; and zone III is the release zone (after Luo and Kitanidis [2004], with permission from Elsevier).

[4] Interestingly, many studies cited above reported a power law behavior of concentration at late time with exponent $-3 / 2$. This exponent is consistent with mass transfer described by a diffusion model [Crank, 1975]. Thus, in order to characterize kinetic mass transfer in the experiments, most researchers applied either the diffusion model or a multirate model [Haggerty and Gorelick, 1995; Haggerty et al., 2001; Widestrand et al., 2007]. In highly heterogeneous systems with kinetic mass transfer it is generally difficult to identify the primary mechanism responsible for the enhanced BTC tailing [Selroos and Cvetkovic, 1992]. Cvetkovic and Haggerty [2002] analyzed this case by weighting the local BTC, related to a certain residence time $\tau$ in the mobile zones, with the probability density of $\tau$ and integrating over all possible values of $\tau$. In this context, the distribution of $\tau$ reflects heterogeneous advection, which may be correlated to the mass exchange between the mobile and stagnant zones. Cvetkovic and Haggerty [2002] found that the traveltime distribution can significantly affect the BTC behavior at late time. More interestingly, their derivation showed that the BTC power law behavior may display different exponents if the traveltime distribution tail also follows a power law. Becker and Shapiro [2000, 2003] observed power law behavior in late-time BTCs of forced-gradient tracer tests in fractured crystalline rock, which they explained by heterogeneous advection.

[5] In the present study, we analyze transport in a dipole flow field created by an extraction-injection well pair with equal pumping strength in homogeneous media. Particularly, we are interested in the BTC tailing behavior in the extraction well. Dipole flow fields have been applied in many field-scale applications for forced-gradient tracer testing and groundwater remediation [e.g., Welty and Gelhar, 1994; Hadermann and Heer, 1996; Sutton et al., 2000; Cunningham and Reinhard, 2002; Novakowski et al., 2004; Ptak et al., 2004; Tiedeman and Hsieh, 2004; Luo et al., 2006a; Wu et al., 2006]. Experimental results showed the late-time BTC behavior in a dipole flow field followed a power law decay, which cannot be characterized by the Gaussian behavior predicted by quasi-one-dimensional ADEs [Kurowski et al., 1994; Hadermann and Heer, 1996; Becker and Shapiro, 2000, 2003]. Muskat [1937] determined the shape and position of a tracer front for a dipole flow field in the absence of regional flow and the advective breakthrough time for the injected tracer reaching the extraction well. Recently, more analytical and semianalytical solutions were also developed to evaluate the traveltime distribution and reactive transport within the recirculation zone between the injection and extraction well in homogeneous and heterogeneous media [Chilakapati and Yabusaki, 1999; Dagan and Indelman, 1999; Zhan, 1999; Cunningham et al., 2004; Luo and Kitanidis, 2004]. However, none of these reported any late-time behavior of the traveltime distribution. Zhang and Koplik [1997] and Koplik [2001] showed that in a dipole flow field in a circular domain the traveltime distribution has a region of power law decay, a transitional shoulder, an exponential decay region. Unlike the research mentioned above, our present work attempts to study the relative importance and effects of different flow and transport mechanisms on the late-time tailing behavior in a dipole flow field. In particular, we want to examine the BTC tailing behavior when both the traveltime distribution and transport mechanisms may be responsible for the power law behavior of late-time BTCs. The paper is organized as follows: section 2 describes the dipole flow field mostly applied in field-scale applications of tracer testing and groundwater remediation; section 3 derives an asymptotic solution at late time to the traveltime distribution in a dipole flow in the absence of regional flow by extending our previous analytical solutions; the impacts of regional flow and dispersion on the traveltime distribution are analyzed in section 4 and 5, respectively; section 6 extend the analysis by studying the combined effect of tracer transport in the dipole flow configuration under diffusive mass transfer and first-order sorption kinetics and analyze the effect on the tailing behavior of BTCs; finally, section 7 summarizes the main results and conclusions.

\section{Dipole Flow Field}

[6] The methodology to study the dipole flow field follows the studies of Luo and Kitanidis [2004] and Luo et al. [2006b], who derived an analytical solution of traveltimes in an ideal dipole flow field in the absence of dispersion and regional flow, and a semianalytical scheme in a dipole flow field in the presence of regional flow to quickly evaluate the traveltime distribution. As illustrated in Figure 1, we consider an extraction and an injection well in a confined homogeneous and isotropic aquifer. The system of coordinates is chosen such that the extraction well is located at $(d, 0)$, and the injection well at $(-d, 0)$. The uniform regional flow is oriented at angle $\alpha$ from the positive $x$ axis. Water is pumped from the extraction well 


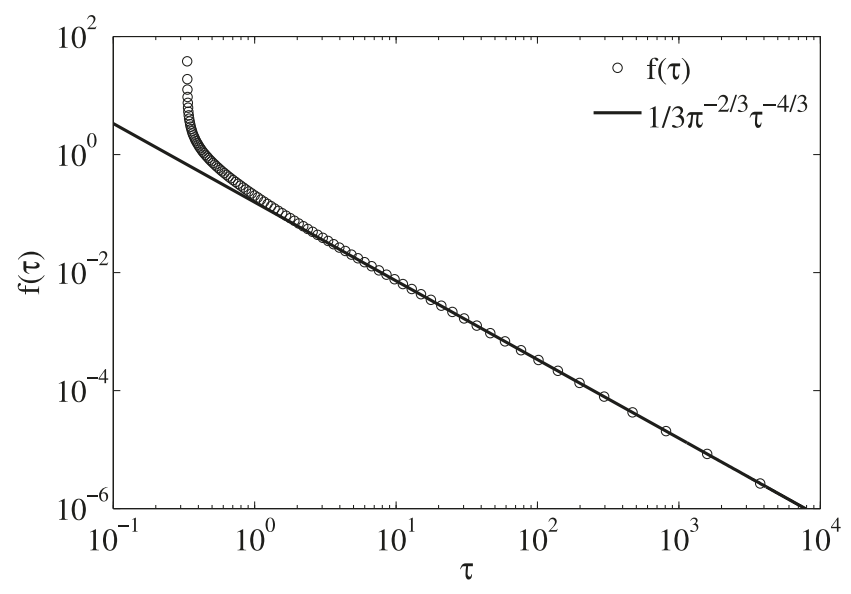

Figure 2. Traveltime distribution from the injection well to the extraction well in an ideal dipole flow in the absence of dispersion and regional flow.

with a pumping rate $Q_{\mathrm{w}}$ and reinjected into the injection well with the same rate. Generally, the created flow field consists of a capture zone, a recirculation zone, and a release zone, delineated by the separating streamlines passing through the stagnation points (denoted zones I, II, and III in Figure 1, respectively). Outside of the bounding streamlines regional flow does not pass through any of the wells. We are interested in the traveltime distribution within the recirculation zone from the injection well to the extraction well (zone II in Figure 1).

\section{Traveltime Tailing in an Ideal Dipole Flow Field}

[7] In an ideal dipole flow field without regional flow, the traveltime distribution at the extraction well caused by advection only is given by (for details, see Luo and Kitanidis [2004])

$$
\tau=\frac{\tau_{\mathrm{d}}}{T}=\frac{1+\pi\left(1 \quad \Psi_{\mathrm{n}}\right) \cot \left(\pi \Psi_{\mathrm{n}}\right)}{\sin ^{2}\left(\pi \Psi_{\mathrm{n}}\right)}
$$

in which $\tau_{\mathrm{d}}$ is the dimensional traveltime, $\tau$ is the dimensionless traveltime, normalized by the characteristic time $T$ (the median traveltime) that depends on the distance $2 d$ between the wells, the pumping rate $Q_{\mathrm{w}}$, the effective porosity $n$, and the thickness of the aquifer $b$ :

$$
T=\frac{4 \pi n b d^{2}}{Q_{\mathrm{w}}}
$$

In the rest of the manuscript, all the time variables without particular definition will be rendered dimensionless by dividing by $T . \Psi_{\mathrm{n}}$ is the dimensionless stream function:

$$
\Psi_{\mathrm{n}}=\frac{2|\Psi|}{Q_{\mathrm{w}}}
$$

where $\Psi$ is given by

$$
\Psi=\frac{Q_{\mathrm{w}}}{2 \pi}\left[\tan ^{-1}\left(\frac{y}{x d}\right) \quad \tan ^{-1}\left(\frac{y}{x+d}\right)\right]
$$

where $x$ and $y$ are spatial coordinates.
[8] Because the flow field is symmetric and the flow rate within a stream tube can be determined by the stream function difference, the cumulative density function $(c d f)$ of $\tau$ is given by [Luo and Kitanidis, 2004]

$$
F(\tau)=1 \quad \Psi_{\mathrm{n}}(\tau)
$$

Substituting (5) into (1) leads to

$$
\tau=\frac{1 \quad \pi F \cot (\pi F)}{\sin ^{2}(\pi F)}
$$

By taking the derivative of equation (6) with respect to $F$, we obtain

$$
\frac{\mathrm{d} \tau}{\mathrm{d} F}=\frac{3 \pi \sin (\pi F) \cos (\pi F)+\pi^{2} F+2 \pi^{2} F \cos ^{2}(\pi F)}{\sin ^{4}(\pi F)}
$$

The traveltime distribution, $\psi_{0}$, is then given by

$$
\psi_{0}(\tau)=\frac{\mathrm{d} F}{\mathrm{~d} \tau}=\frac{\sin ^{4}(\pi F)}{3 \pi \sin (\pi F) \cos (\pi F)+\pi^{2} F+2 \pi^{2} F \cos ^{2}(\pi F)}
$$

where $F$ is calculated by equation (6) for a given $\tau$.

[9] At late time, i.e., $F \rightarrow 1$, (6) can be approximated by

$$
\tau \cong \frac{\pi \quad \pi F+\pi F}{(\pi \quad \pi F)^{3}}=\pi^{-2}(1 \quad F)^{-3}
$$

resulting in

$$
F=1 \quad \pi^{-2 / 3} \tau^{-1 / 3}
$$

Taking the derivative of equation (10) with respect to $\tau$, we obtain that the late-time behavior of the traveltime distribution at the extraction well follows a power law with an exponent of $-4 / 3$ :

$$
\psi_{0}=\frac{1}{3} \pi^{-2 / 3} \tau^{-4 / 3}
$$

The power law behavior is consistent to that reported by Koplik et al. [1994], in which they described the traveltime as a function of the streamline orientation at the injection well.

[10] Figure 2 shows the traveltime distribution at the extraction well in a log-log plot. After a short initial time, $\psi_{0}$ becomes a straight line with a slope of $k=-4 / 3$. The asymptotic solution of equation (11) matches the late-time distribution. Because the power law exponent is larger than -2 , the first-order moment of the traveltime distribution is infinite. That is, a mean traveltime cannot be defined in the ideal dipole flow field.

\section{Traveltime Tailing in the Presence of Regional Flow}

[11] As already mentioned, in the presence of uniform regional flow, the flow field generally consists of a capture zone, a recirculation zone, a release zone, and a regional 

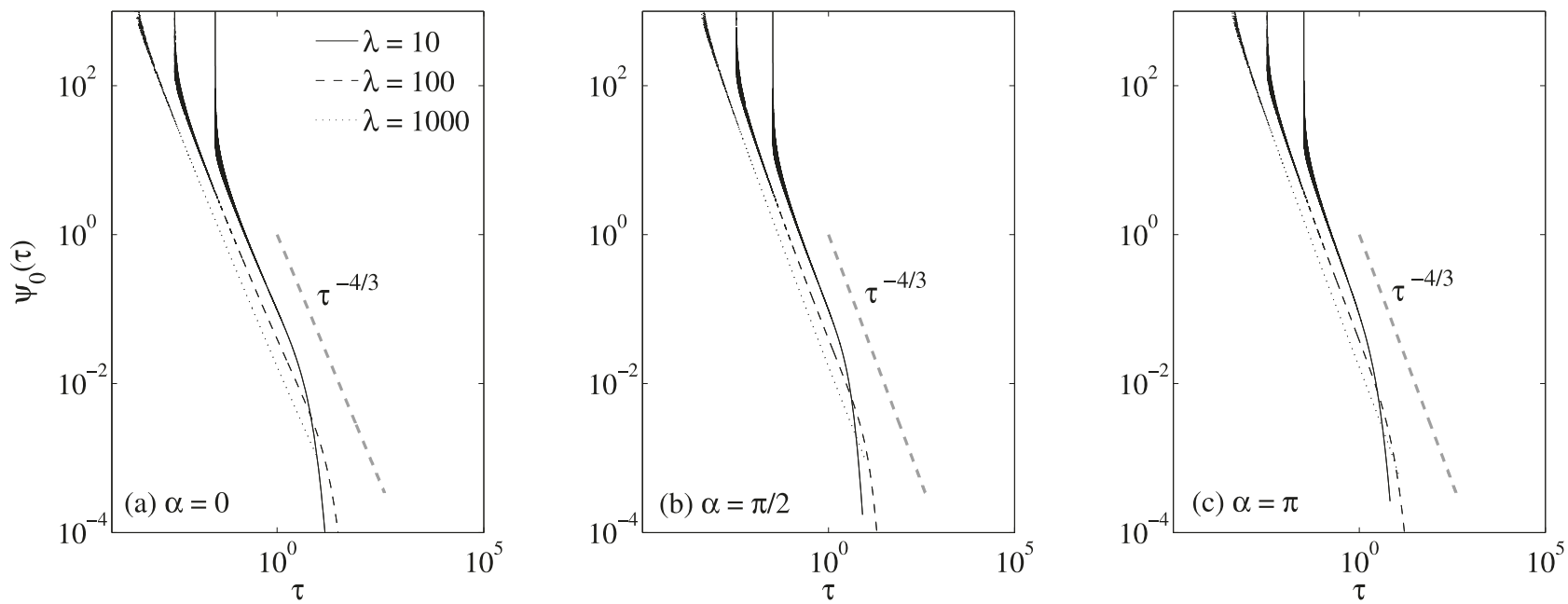

Figure 3. Effects of well pumping rates on the traveltime distribution in the dipole flow field with regional flow; $\alpha=0$ indicates that the regional flow is exactly in the direction from the injection well to the extraction well; $\alpha=\pi / 2$ indicates that the regional flow is perpendicular to the well placement; and $\alpha=\pi$ indicates that the regional flow is exactly from the extraction well to the injection well.

flow zone that is not connected to any of the pumping wells. However, these zones do not always coexist. For example, when $\alpha=0$, there is only a recirculation zone, but there are no capture and release zones. For $\alpha \neq 0$, a critical pumping rate must be exceeded to maintain recirculation. Luo et al. [2006b] identified this critical value in dimensionless terms. Considering the specific discharge $q_{0}$ of regional flow, Luo et al. [2006b] identified a minimal dimensionless pumping rate $\lambda=Q_{w} / 2 \pi d q_{0}$ of 0.6366 , guaranteeing that a recirculation zone exists regardless of the orientation of regional flow. In the following, we consider only cases where this condition is satisfied. We evaluate traveltimes from the injection well to the extraction well by a streamline tracing technique using a fourth-order Runge-Kutta scheme based on the complex discharge function, which was developed for capture zone delineation [Fienen et al., 2005]. The number of traced streamlines is 36,000 .

[12] Figure 3 shows the effects of the well pumping rate and the regional flow orientation on the late-time behavior of the BTC. Here, the dimensionless traveltime $\tau$ is defined by $\tau=\tau_{\mathrm{d}} / T_{0}^{*}$, in which $T_{0}^{*}=2 n b d / q_{0}$ is the time needed by a solute particle to travel the distance between the two wells exclusively by regional flow. The thick grey lines are reference lines with a power law exponent of $-4 / 3$. The traveltime distributions follow a power law decay with exponent $-4 / 3$ for a limited time period, followed by exponential decay behavior. The results indicate that higher the pumping rate, the longer the period with power law behavior. The exponential behavior ensures that the first and all higher moments of the traveltime distribution are finite, which corresponds to a recirculation zone with finite area. The case $\alpha=0$ represents the situation where the regional flow is oriented exactly in the direction from the injection to the extraction well, creating a completely closed recirculation zone, whereas $\alpha=\pi$ represents the case where regional flow is oriented exactly from the extraction to the injection well, creating a large capture zone. The late-time behavior of the traveltime distribution is similar for all orientations. In addition, a larger regional flow angle means that the exponential behavior begins earlier. That is, for a given pumping rate, the power law behavior lasts the longest when $\alpha=0$. Furthermore, at high pumping rates, the early time residence time distribution are not affected by the regional flow orientation; that is, only the late-time behavior is influenced by the well placement (or regional flow orientation) at large well pumping rates.

\section{Effects of Dispersion}

[13] Dispersion modifies traveltime distributions. We first consider only longitudinal dispersion which does not change the travel paths of particles; that is, the particles still move along streamlines. We evaluate the travel times by a particle tracking/random walk scheme, corrected by the stream function [Strack, 1989]. In the presence of transverse dispersion, particles are allowed to cross the streamlines. The spatial discretization is $\Delta s=0.002$ (a dimensionless spatial step normalized by $d$ ), and we apply the fourth-order Runge-Kutta scheme with corrected velocity field in the vicinity of the wells [Kitanidis, 1994; Zheng and Bennett, 2002]. 36,000 particles are initially distributed at the injection-well boundary according to the discharge function.

[14] Figure 4 shows the residence time distributions at the extraction well in the presence of local dispersion, but in the absence of regional flow. Local dispersion significantly influences the residence time distributions at early times. In particular, longitudinal dispersion enhances and transverse dispersion diminishes spreading of the BTCs. However, local dispersion does not change the power law decay behavior at large times. This characteristics was identified by Welty and Gelhar [1994], in which only the early time tracer BTC was used to approximate the dispersion coefficient. Because of the numerical random walk approach, the distributions shown in Figure 4 are not as smooth as those computed for strictly advective transport. Nonetheless, they all show the same power law behavior with an exponent of $-4 / 3$. In fact, local dispersion cannot change the fact of an infinite recirculation zone, so that the mean residence time remains infinite. The distribution of the streamlines, differ- 

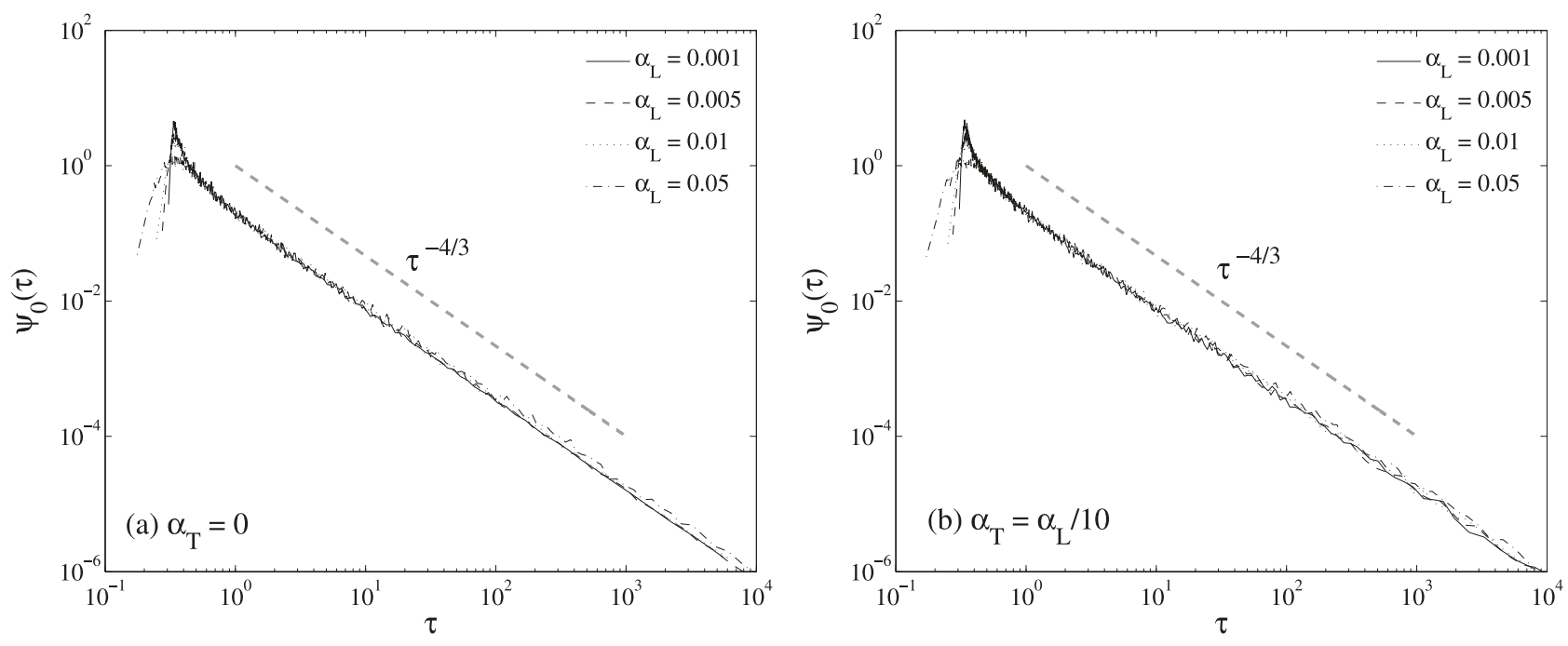

Figure 4. Effects of local dispersion on the traveltime distribution in the dipole flow field in the absence of regional flow. Dispersivities are normalized by the half distance between the two wells. The longitudinal dispersivity is $\alpha_{L}=0.01$, and $\alpha_{T} / \alpha_{L}=10$.

ing in advective traveltime, dominates the tailing behavior of the residence time distribution at the extraction well.

[15] In the presence of both local dispersion and regional flow, the tails of these curves are similar to those obtained for strictly advective transport in a dipole flow field with regional flow. The late-time behavior is dominated by the distribution of streamlines, exhibiting a power law with exponent $-4 / 3$ over a certain time period and changing to exponential decay of the tail at very late times.

\section{Breakthrough Tailing of Tracers Undergoing Linear Mass Transfer}

[16] The above analyses consider either pure advection or advection plus local dispersion. In the presence of an immobile pore space, the mass transfer between the mobile and immobile domains will change the overall residence time distribution. In this context, it is worth noting that firstorder kinetic sorption models and linear mass transfer models are formally equivalent: the immobile/sorbed solute fraction $c_{\text {im }}$ depends linearly on the mobile/dissolved solute fraction $c_{\mathrm{m}}$ [e.g., Carrera et al., 1998; Haggerty et al., 2000; Dentz and Berkowitz, 2003]:

$$
c_{\mathrm{im}}(\mathbf{x}, t)=\int_{0}^{t} \varphi\left(t \quad t^{\prime}\right) c_{\mathrm{m}}\left(\mathbf{x}, t^{\prime}\right) \mathrm{d} t^{\prime}
$$

in which we assume that there is initially no immobilized solute, and $\varphi(t)$ is the so-called memory function. Dentz and Berkowitz [2003] give the following general expression for $\varphi(t)$ :

$$
\varphi(t)=\int_{0}^{\infty} \omega\left(\tau_{\mathrm{im}}\right) \mathcal{P}\left(\tau_{\mathrm{im}}\right) \phi\left(t / \tau_{\mathrm{im}}\right) \mathrm{d} \tau_{\mathrm{im}}
$$

where $\tau_{\text {im }}$ denotes the residence time in an immobile region, $\omega\left(\tau_{\text {im }}\right)$ is the trapping rate, and $\mathcal{P}\left(\tau_{\text {im }}\right)$ is the distribution of residence times. The trapping rate $\omega\left(\tau_{\text {im }}\right)$ and the local memory function $\phi\left(t / \tau_{\text {im }}\right)$ are determined by the particular mass transfer mechanism under consideration. In the following we focus on single-rate diffusive mass transfer and first-order kinetic sorption reaction, i.e.,

$$
\mathcal{P}\left(\tau_{\text {im }}\right)=\delta\left(\tau_{\text {im }} \quad \tau_{0}\right)
$$

in which $\tau_{0}$ is the diffusion time in the immobile zone in the case of diffusive mass transfer and the sorption time for kinetic sorption reactions, and $\delta$ is the delta function. The trapping rate is given by $\omega=\theta \tau_{\text {im }}{ }^{1}$, where $\theta$ is the volume ratio between the mobile and immobile region. The memory function, equation (13), then simplifies to

$$
\varphi(t)=\frac{\theta}{\tau_{0}} \phi\left(t / \tau_{0}\right)
$$

For the following asymptotic analysis, it is convenient to transform the memory function to Laplace space. The Laplace transform is defined in, e.g., Abramowitz and Stegun [1972]; Laplace transformed quantities in the following are denoted by an upper bar. Thus the Laplace transform of the memory function $\varphi(t)$ is given by

$$
\varphi(s)=\theta \phi\left(s \tau_{0}\right)
$$

in which $s$ is the coordinate in the Laplace domain.

\subsection{Traveltime Distribution}

[17] Within this framework, mass conservation gives for the traveltime $\psi(t, \tau)$ on a given streamline:

$$
\frac{\partial \psi(t, \tau)}{\partial t}+\frac{\partial}{\partial t} \int_{0}^{t} \varphi\left(t \quad t^{\prime}\right) \psi\left(t^{\prime}, \tau\right) \mathrm{d} t^{\prime}+\frac{\partial \psi(t, \tau)}{\partial \tau}=0
$$

It may be worth noting that here the traveltime distribution is identical to the flux concentration for an instantaneous unit pulse because local dispersion is neglected. 


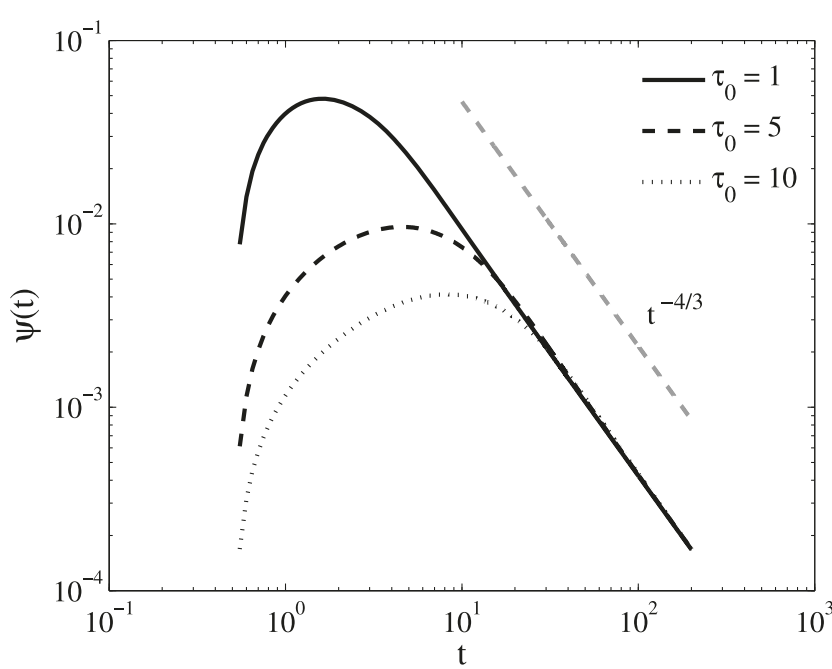

Figure 5. Breakthrough curve of tracers undergoing linear first-order sorption; $\tau_{0}$ is the sorption timescale, normalized by the characteristic time $T$. The gray line is a reference line with exponent $-4 / 3$.

[18] We obtain for the Laplace transform $\psi(s, \tau)$ of $\psi(t, \tau)$ :

$$
s[1+\varphi(s)] \psi(s, \tau)+\frac{\partial \psi(s, \tau)}{\partial \tau}=0
$$

Equation (18) can be integrated straightforwardly to

$$
\psi(s, \tau)=\exp \{s \tau[1+\varphi(s)]\}
$$

A similar expression was derived by Cvetkovic and Haggerty [2002].

[19] Finally, we obtain the Laplace transform of the global traveltime distribution by a weighted integral of equation (19) over all traveltimes:

$$
\psi(s)=\int_{0}^{\infty} \exp \{\quad s \tau[1+\varphi(s)]\} \psi_{0}(\tau) \mathrm{d} \tau
$$

The right side of equation (20) is the Laplace transform of $\psi_{0}(\tau)$ so that it can be written as

$$
\psi(s)=\psi_{0}\{s[1+\varphi(s)]\}
$$

The given form of the traveltime distribution $\psi(s)$ in terms of the corresponding distribution $\psi_{0}(s)$ without mass transfer has been obtained by Sardin et al. [1991], Cvetkovic and Haggerty [2002], and Margolin et al. [2003] in the context of the continuous time random walk method. Equation (21) is the basis for the following asymptotic analysis of the late-time arrival-time distribution considering linear mass transfer in a diploe flow field.

\subsection{Asymptotic Analysis}

[20] In general, we apply the asymptotic analysis of Margolin et al. [2003]. The late-time behavior of the traveltime distribution $\psi_{0}(\tau)$ is given by equation (11) for $\tau \gg T$ in which $T$ is the characteristic time defined by equation (2). Thus the Laplace transform of $\psi_{0}(\tau)$ can be approximated for $s \ll T^{1}$ by [e.g., Dentz and Berkowitz, 2003]

$$
\psi_{0}(s)=1 \quad \frac{T \Gamma(2 / 3)}{\pi^{2 / 3}}(s T)^{1 / 3}+\ldots
$$

Thus, for $s \ll T^{1}, \psi(s)$, equation (21) can be approximated by

$$
\psi(s)=1 \quad \frac{T \Gamma(2 / 3)}{\pi^{2 / 3}}[s T+s T \varphi(s)]^{1 / 3}+\ldots
$$

For $\psi_{0}(t)$, the asymptotic regime is determined by the characteristic time $T$, equation (2); that is, the asymptotic behavior given by equation (11) is valid for traveltimes larger than $T$. For $\psi(t)$, identifying the onset of the asymptotic regime is more complicated, because the memory function $\varphi(t)$ introduces additional timescales that need to be taken into account in the asymptotic analysis. In the following, we study the asymptotic behavior of $\psi(t)$ for transport considering (1) linear driving-force kinetics of sorption and (2) diffusive mass transfer into spheres.

\subsubsection{First-Order Kinetics of Sorption}

[21] For linear first-order sorption, the local memory function is given by

$$
\phi\left(t / \tau_{0}\right)=\exp \left(\frac{t}{\tau_{0}}\right)
$$

The Laplace transform of $\phi(t)$ is given by

$$
\phi(s)=\frac{1}{1+s}
$$

Substituting equation (25) into (16), we obtain for singlerate kinetic sorption

$$
\varphi(s)=\frac{1}{1+s \tau_{0}}
$$

For times large compared to the typical adsorption time $\tau_{0}$, which corresponds to $s \ll \tau_{0}{ }^{1}$, equation (26) can be expanded in $s$ according to

$$
\varphi(s)=1 \quad s \tau_{0}+\ldots
$$

Substituting equation (27) into equation (20), using equation (23) and subsequent expansion for $s \ll \min \left(\tau_{0}{ }^{1}\right.$, $T^{1}$ ) gives for $\psi(s)$ in leading order:

$$
\psi(s)=1 \quad \frac{T \Gamma(2 / 3)}{\pi^{2 / 3}}(s T)^{1 / 3}\left(1+s \tau_{0}\right)^{1 / 3}+\ldots
$$

which is in leading order identical to equation (22). Thus, asymptotically, the tailing behavior is the same as in the case without sorption. Figure 5 shows the adsorption timescale $\tau_{0}$ can only affect the time for the breakthrough tails to start the power law behavior, but cannot change the exponent $-4 / 3$, which is fully controlled by the traveltime distribution. The behavior is more interesting in the case of diffusive mass transfer studied in the following. 


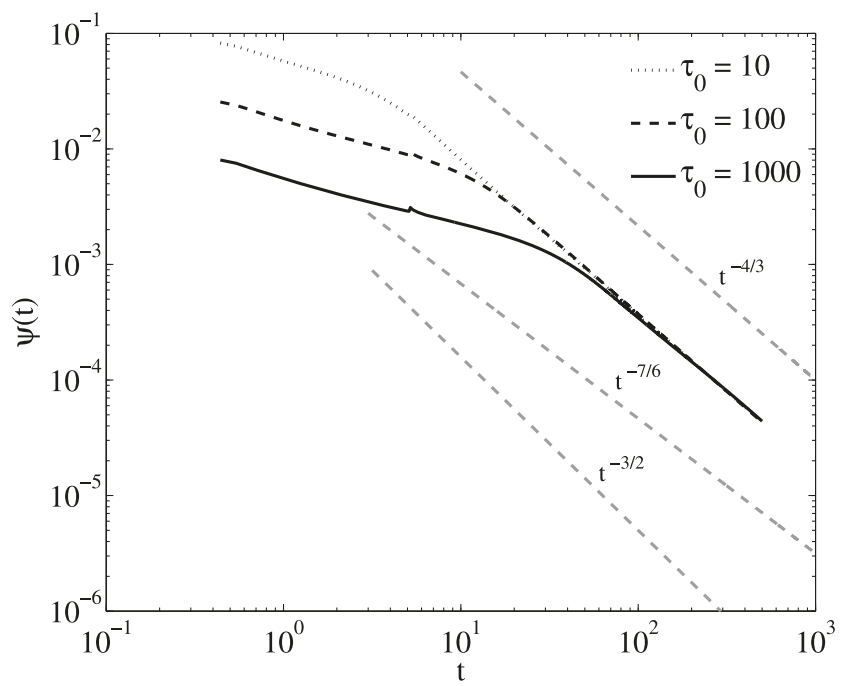

Figure 6. Breakthrough curve of tracers undergoing diffusive mass transfer into spherical immobile zones; $\tau_{0}$ is the diffusion timescale, normalized by the characteristic time $T$. The gray lines are reference lines with exponents $-4 / 3,-7 / 6$, and $-3 / 2$.

\subsubsection{Diffusive Mass Transfer Into Spheres}

[22] For diffusive mass transfer into spherical immobile zones the memory function $\phi\left(t / \tau_{\text {im }}\right)$ is defined by its Laplace transform as [e.g., Harvey and Gorelick, 1995]

$$
\phi(s)=\frac{1}{\sqrt{ } s}\left[\begin{array}{ll}
\operatorname{coth}(\sqrt{ } s) & \frac{1}{\sqrt{ } s}
\end{array}\right]
$$

[23] Substituting equation (29) into equation (16) and equation (25) into equation (13), we obtain for the memory function for single rate diffusive mass transfer

$$
\varphi(s)=\frac{\theta}{\sqrt{ } s \tau_{0}}\left[\begin{array}{ll}
\operatorname{coth}\left(\sqrt{ } s \tau_{0}\right) & \frac{1}{\sqrt{ } s \tau_{0}}
\end{array}\right]
$$

For times small compared to the typical diffusion time $\tau_{0}$ in the immobile spheres, $t \ll \tau_{0}$, which corresponds to $s \gg$ $\tau_{0}{ }^{1}$, the memory function can be expanded in $s$ according to

$$
\varphi(s)=\frac{\theta}{\sqrt{ } s \tau_{0}}+\ldots
$$

where the dots denote subleading contributions. For traveltimes much larger than the diffusion timescale $\tau_{0}$; that is, for $s \ll \tau_{0}{ }^{1}$, the memory function behaves as

$$
\varphi(s)=\frac{\theta}{3}\left(\begin{array}{ll}
1 & \frac{s}{15}
\end{array}\right)+\ldots
$$

where the dots denote higher-order terms contributions.

[24] We assume that the characteristic timescale $T$ and the diffusion timescale $\tau_{0}$ are well separated, $T \ll \tau_{0}$. Then, $T$ and $\tau_{0}$ define two relevant time regimes. (1) the intermediate time regime $T \ll t \ll \tau_{0}$. Note that for diffusive mass transfer in uniform flow the BTC behaves as $t^{3 / 2}$ in this time regime [e.g., Hadermann and Heer, 1996]. (2) The asymptotic large-time regime $t \gg \tau_{0}$. In the intermediate time regime, which corresponds to $\tau_{0}{ }^{1} \ll s \ll T^{1}$ for the Laplace variable, the Laplace transformed traveltime distribution $\psi_{0}(s)$ can be approximated by equation (23) while the Laplace transformed memory function $\psi(s)$ is given by equation (32). Thus we obtain for $\psi(s),(21)$, in this regime

$$
\psi(s)=1 \quad \frac{T^{4 / 3} \theta^{1 / 3} \Gamma(2 / 3)}{\tau_{0}^{1 / 6} \pi^{2 / 3}} s^{1 / 6}+\ldots
$$

where the dots denote subleading contributions. Inversion of equation (33) [e.g., Dentz and Berkowitz, 2003] gives the power law behavior

$$
\psi(t)=A\left(\frac{t}{T}\right)^{-7 / 6}+\ldots
$$

for $\psi(t)$ in the intermediate time regime $T \ll t \ll \tau_{0}$. The constant $A$ is defined by

$$
A=\frac{\theta^{1 / 3} \Gamma(2 / 3)}{6 \Gamma(5 / 6) \pi^{2 / 3}}\left(\frac{T}{\tau_{0}}\right)^{1 / 6}
$$

Thus the tailing behavior of the traveltime distribution $\psi_{0}(t)$ can be changed in the presence of diffusive mass transfer mechanisms and lead to a tail characterized by the exponent $-7 / 6$ if the characteristic time $T$ and the diffusion timescale $\tau_{0}$ are well separated.

[25] The above analyses can be conveniently extended to generalize the case of power law traveltime distribution and power law mass transfer (either diffusive or sorptive). Consider the traveltime distribution with asymptotic latetime behavior $\psi_{0}(t) \sim t^{\gamma}$ [Berkowitz et al., 2006] and a memory function with asymptotic behavior $\varphi(t) \sim t^{\beta}$ [Haggerty et al., 2000] in the time regime $T \ll t \ll \tau_{0}$. The resulting asymptotic power law behavior on this timescale of combining the traveltime distribution and the memory function is

$$
\psi(t) \propto t^{-1-\beta(\gamma-1)}, \text { for } 0<\beta<1,1<\gamma<2
$$

[26] In the late-time regime $t \gg \tau_{0}$, i.e., $s \ll \tau_{0}{ }^{1}$, the Laplace transform of the traveltime distribution is obtained by substituting equation (32) into equation (23):

$$
\psi(s)=1 \quad \frac{T \Gamma(2 / 3)}{\pi^{2 / 3}}\left(1+\frac{\theta}{3}\right)(s T)^{1 / 3}+\ldots
$$

The inverse Laplace transform of equation (37) gives for the travel time distribution in the long-time regime $t \gg \tau_{0}$

$$
\psi(t)=B\left(\frac{t}{T}\right)^{-4 / 3}
$$

with the constant $B$ defined by

$$
B=\frac{1+\theta / 3}{3 \pi^{2 / 3}}
$$

Thus the long-time tailing behavior of the traveltime distribution remains unchanged in the presence of diffusive mass transfer. 
[27] Figure 6 shows the BTC tailing with different diffusive timescales. For small $\tau_{0}$, the intermediate regime with exponent $-7 / 6$ is very short. With the increase of $\tau_{0}$, the intermediate regime becomes longer and more obvious. At very large time, $t \gg \tau_{0}$, all the BTCs fall into a same line with exponent $-4 / 3$. Note, however, that in practical applications the diffusion time $\tau_{0}$ can be very large and so that it can take very long until this asymptotic time regime is reached. In the more relevant intermediate regime, in contrast, the tailing behavior is changed, as outlined above. Note that the lines with different power law exponents (the grey lines in Figure 6) are not dramatically different. In practical applications, accurate measurements at low concentrations must be taken in order to analyze the controlling mechanisms responsible for the power law tailing behavior.

\section{Conclusions}

[28] The distribution of traveltime from the injection to the extraction well in a dipole flow field has interesting latetime behavior. In an ideal dipole flow field in homogeneous media in the absence of regional flow, the traveltime distribution, $\psi_{0}(\tau)$, follows a power law with exponent $-4 / 3$, which also occurs at large well pumping rates with regional flow. This behavior is not surprising given that the recirculation area is infinite. In the case with regional flow, which creates a finite recirculation area, $\psi_{0}$ shows a power law with exponent $-4 / 3$ for a certain period of time, followed by exponential decay, which assures a finite mean value, as well as finite higher-order moments of the traveltime distribution. Local dispersion is found not to influence the late-time behavior, but has significant effects at early times. Thus the early time BTC is important for estimating dispersion coefficients.

[29] BTCs at the extraction well in a dipole flow field are highly affected by the nonuniform distribution of travel paths and the nonuniform velocity field, resulting in different traveltimes along the streamlines. We analyzed the traveltime distribution $\psi(t)$ for tracers undergoing diffusive mass transfer from mobile into immobile regions and first-order sorption kinetic. In both cases the immobile/adsorbed solute fraction is given by a linear functional of the mobile/ nonadsorbed solute fraction. We consider single linear kinetic sorption, which is characterized by an exponential memory function. In this case the tailing behavior is controlled by the dipole flow geometry implying the exponent $-4 / 3$. For diffusive mass transfer, we distinguish two relevant time regimes determined by the typical diffusion time $\tau_{0}$ in the immobile regions and the characteristic breakthrough time without mass transfer, $T$, which are assumed to be well separated, $T \ll \tau_{0}$. In the intermediate time regime $T \ll t \ll \tau_{0}$, the breakthrough tailing is characterized by the exponent $-7 / 6$ as opposed to the $-4 / 3$ without mass transfer. The exponent $-7 / 6$ is given by the characteristic exponent $\beta=1 / 2$ for the memory function of matrix diffusion and the exponent $\gamma=4 / 3$ determined by the dipole flow geometry, as $-1-\beta(\gamma-1)$, which is the generalized result for the case of power law traveltime distribution and power law mass transfer. In the long-time regime $t \gg \tau_{0}$ the exponent by which the BTC decays is again $-4 / 3$ as given by the dipole flow geometry. Thus, in an ideal dipole flow field in homogeneous media in the absence of regional flow, the BTC tailing behavior is ultimately dominated by the nonuniform flow field over dispersion, sorption, or mass transfer. In the presence of sufficiently strong regional flow, yet another regime may occur, where the advective traveltime distribution, $\psi_{0}(\tau)$ changes to exponential tailing behavior before the diffusive timescale is reached. This would result in power law tailing dominated by diffusive mass transfer, i.e., with exponent $-3 / 2$.

[30] Our study exemplifies that the flow configuration has significant impact on the tailing behavior of BTCs. Power law tailing can be caused by (1) kinetic mass transfer, (2) variability of hydraulic conductivity, and (3) flow configuration. If the tailing of BTCs obtained by well-to-well tracer tests is used to identify mechanisms of kinetic mass transfer, the impact of the flow configuration must not be neglected. It may be necessary to observe the breakthrough curve for very long periods of time in order to identify the various regimes involved. Obviously, this requires accurate measurements at low concentrations. Premature truncation of the test, which may be enforced by reaching the limit of tracer detection, may lead to erroneous interpretations regarding mass transfer kinetics. In addition, in heterogeneous media, most flow may travel along preferential flow paths, resulting in fast decay of the actual traveltime distribution, and the latetime behavior of the BTC may be ultimately dominated by kinetic mass transfer.

[31] Acknowledgments. This material is based upon work supported by the National Science Foundation under grant NSF/EAR 0001441-002, by the United States Department of Energy (DOE) Environmental Remediation Sciences Division (ERSD) Biological and Environmental Research (BER) grant DE-F603-00ER63046, and by the program "Ramon y Cajal" and the project MODEST (project CGL-2005-05171) of the Spanish Ministry of Education and Science. This article has not been reviewed by the agency, and no official endorsement should be inferred. Any opinions, findings, and conclusions or recommendations expressed in this material are those of the authors and do not necessarily reflect the views of NSF and DOE. We would like to thank R. Haggerty, J. Carrera, and two anonymous reviewers for their constructive comments to this manuscript.

\section{References}

Abramowitz, M., and I. A. Stegun (1972), Handbook of Mathematical Functions with Formulas, Dover, New York.

Becker, M. W., and A. M. Shapiro (2000), Tracer transport in fractured crystalline rock: Evidence of nondiffusive breakthrough tailing, Water Resour. Res., 36, 16771686.

Becker, M. W., and A. M. Shapiro (2003), Interpreting tracer breakthrough tailing from different forced-gradient tracer experiment configurations in fractured bedrock, Water Resour. Res., 39(1), 1024, doi:10.1029/ 2001WR001190.

Berkowitz, B., and H. Scher (1997), Anomalous transport in random fracture networks, Phys. Rev. Lett., 79, 40384041.

Berkowitz, B., and H. Scher (1998), Theory of anomalous chemical transport in random fracture networks, Phys. Rev. E, 57, 58585869.

Berkowitz, B., H. Scher, and S. E. Silliman (2000), Anomalous transport in laboratory-scale, heterogeneous porous media, Water Resour. Res., 36, 149158.

Berkowitz, B., A. Cortis, M. Dentz, and H. Scher (2006), Modeling nonFickian transport in geological formations as a continuous time random walk, Rev. Geophys., 44, RG2003, doi:10.1029/2005RG000178.

Carrera, J., X. Sanchez-Vila, I. Benet, A. Medina, G. Galarza, and J. Guimera (1998), On matrix diffusion: Formulations, solution methods and qualitative effects, Hydrogeol. J., 6, 178190.

Chilakapati, A., and S. Yabusaki (1999), Nonlinear reactions and nonuniform flows, Water Resour. Res., 35, 24272438.

Crank, J. (1975), The Mathematics of Diffusion, Oxford Univ. Press, New York.

Cunningham, J. A., and M. Reinhard (2002), Injection-extraction treatment well pairs: An alternative to permeable reactive barriers, Ground Water, 40, 599607. 
Cunningham, J. A., T. P. Hoelen, G. D. Hopkins, C. A. Lebron, and M. Reinhard (2004), Hydraulics of recirculating well pairs for ground water remediation, Ground Water, 42, 880889.

Cvetkovic, V., and R. Haggerty (2002), Transport with multiple-rate exchange in disordered media, Phys. Rev., 65, 051308, doi:10.1103/ PhysRevE.65.051308.

Dagan, G., and P. Indelman (1999), Reactive solute transport inflow between a recharging and a pumping well in a heterogeneous aquifer, Water Resour. Res., 35, 36393647.

Dentz, M., and B. Berkowitz (2003), Transport behavior of a passive solute in continuous time random walks and multirate mass transfer, Water Resour. Res., 39(5), 1111, doi:10.1029/2001WR001163.

Dentz, M., A. Cortis, and B. Berkowitz (2004), Time behavior of solute transport in heterogeneous media: Transition from anomalous to normal transport, Adv. Water Resour., 27, 155173.

Di Donato, G., and M. J. Blunt (2004), Streamline-based dual-porosity simulation of reactive transport and flow in fractured reservoirs, Water Resour. Res., 40, W04203, doi:10.1029/2003WR002772.

Di Donato, G., E. Obi, and M. J. Blunt (2003), Anomalous transport in heterogeneous media demonstrated by streamline-based simulation, Geophys. Res. Lett., 30(12), 1608, doi:10.1029/2003GL017196.

Dunnivant, F. M., P. M. Jardine, D. L. Taylor, and J. F. McCarthy (1992), Transport of naturally-occurring dissolved organic-carbon in laboratory columns containing aquifer material, Soil Sci. Soc. Am. J., 56, 437444.

Farrell, J., and M. Reinhard (1994), Desorption of halogenated organics from model solids, sediments, and soil under unsaturated conditions, 2 , Kinetics, Environ. Sci. Technol., 28, 6372.

Fienen, M. N., J. Luo, and P. K. Kitanidis (2005), Semi-analytical, homogeneous, anisotropic capture zone delineation, J. Hydrol., 312, 3950.

Fiori, A., I. Jankovic, and G. Dagan (2006), Modeling flow and transport in highly heterogeneous three-dimensional aquifers: Ergodicity, Gaussianity, and anomalous behavior 2. Approximate semianalytical solution, Water Resour. Res., 42, W06D13, doi:10.1029/2005WR004752.

Guswa, A. J., and D. L. Freyberg (2000), Slow advection and diffusion through low permeability inclusions, J. Contam. Hydrol., 46, 205232.

Hadermann, J., and W. Heer (1996), The Grimsel (Switzerland) migraton experiment: Integrating field experiments, laboratory investigations and modelling, J. Contam. Hydrol., 21, 87100.

Haggerty, R., and S. M. Gorelick (1995), Multiple-rate mass transfer for modeling diffusion and surface reactions in media with pore-scale heterogeneity, Water Resour. Res., 31, 23832400.

Haggerty, R., S. A. McKenna, and L. C. Meigs (2000), On the late-time behavior of tracer test breakthrough curves, Water Resour. Res., 36, 34673479 .

Haggerty, R., S. W. Fleming, and L. C. Meigs (2001), Tracer tests in a fractured dolomite: 2 . Analysis of mass transfer in single-well injectionwithdrawal tests, Water Resour. Res., 37, 11291142.

Harvey, C. F., and S. M. Gorelick (1995), Temporal moment-generating equations: Modeling transport and mass transfer in heterogeneous aquifers, Water Resour. Res., 31, 18951911.

Kitanidis, P. K. (1994), Particle-tracking equation for the solution of the advection-dispersion equation with variable coefficients, Water Resour. Res., 30, 32253227.

Koplik, J. (2001), The tracer transit-time tail in multipole reservoir flows, Trans. Porous Media, 42, 199209.

Koplik, J., S. Redner, and E. J. Hinch (1994), Tracer dispersion in planar multipole flows, Phys. Rev. E, 50, 46504671.

Kurowski, P., I. Ippolito, J. P. Hulin, J. Koplik, and E. J. Hinch (1994), Anomalous-dispersion in a dipole flow geometry, Phys. Fluids, 6, 108 117.

Levy, M., and B. Berkowitz (2003), Measurement and analysis of nonFickian dispersion in heterogeneous porous media, J. Contam. Hydrol., 64, 203226.

Luo, J., and P. K. Kitanidis (2004), Fluid residence times within a recirculation zone created by an extraction-injection well pair, J. Hydrol., 295, 149162.

Luo, J., O. A. Cirpka, M. N. Fienen, W.-M. Wu, T. L. Mehlhorn, J. Carley, P. M. Jardine, C. S. Criddle, and P. K. Kitanidis (2006a), A parametric transfer function methodology for analyzing reactive transport in nonuniform flow, J. Contam. Hydrol., 83, 2741.

Luo, J., W.-M. Wu, M. N. Fienen, P. M. Jardine, T. L. Mehlhorn, D. B. Watson, O. A. Cirpka, C. S. Criddle, and P. K. Kitanidis (2006b), A nested-cell approach for in situ remediation, Ground Water, 44, 266 274.

Margolin, G., M. Dentz, and B. Berkowitz (2003), Continuous time random walk and multirate mass transfer modeling of sorption, Chem. Phys., 295, 7180 .

Muskat, M. (1937), The Flow of Homogeneous Fluids Through Porous Media, McGraw-Hill, New York.

Novakowski, K. S., G. Bickerton, and P. Lapcevic (2004), Interpretation of injection-withdrawal tracer experiments conducted between two wells in a large single fracture, J. Contam. Hydrol., 73, 227247.

Ptak, T., M. Piepenbrink, and E. Martac (2004), Tracer tests for the investigation of heterogeneous porous media and stochastic modelling of flow and transport A review of some recent developments, J. Hydrol., 294, 122163.

Sardin, M., D. Schweich, F. J. Leij, and M. T. van Genuchten (1991), Modeling the nonequilibrium transport of linearly interacting solutes in porous media: A review, Water Resour. Res., 27, 22872307.

Selroos, J. O., and V. Cvetkovic (1992), Modeling solute advection coupled with sorption kinetics in heterogeneous formations, Water Resour. Res., 28,12711278

Strack, O. D. L. (1989), Groundwater Mechanics, Prentice-Hall, Englewood Cliffs, N. J.

Sutton, D. J., Z. J. Kabala, D. E. Schaad, and N. C. Ruud (2000), The dipole-flow test with a tracer, a new single-borehole tracer test for aquifer characterization, J. Contam. Hydrol., 44, 71101.

Tiedeman, C. R., and P. A. Hsieh (2004), Evaluation of longitudinal dispersivity estimates from simulated forced- and natural-gradient tracer tests in heterogeneous aquifers, Water Resour. Res., 40, W01512, doi:10.1029/2003WR002401.

Welty, C., and L. W. Gelhar (1994), Evaluation of longitudinal dispersivity from nonuniform flow tracer tests, J. Hydrol., 153, 71 102.

Werth, C. J., J. A. Cunningham, P. V. Roberts, and M. Reinhard (1997), Effects of grain-scale mass transfer on the transport of volatile organics through sediments: 2. Column results, Water Resour. Res., 33, 2727 2740 .

Widestrand, H., J. Byegård, V. Cvetkovic, E.-L. Tullborg, A. Winberg, P. Andersson, and M. Siitari-Kauppi (2007), Sorbing tracer experiments in a crystalline rock fracture at Äspö (Sweden): 1. Experimental setup and microscale characterization of retention properties, Water Resour. Res., doi:10.1029/2006WR005277, in press.

Wu, W.-M., et al. (2006), Pilot-scale in situ bioremediation of uranium in a highly contaminated aquifer. 2. Reduction of U(VI) and geochemical control of U(VI) bioavailability, Environ. Sci. Technol., 40, 39863995.

Zhan, H. (1999), Analytical and numerical modeling of a double well capture zone, Math. Geol., 31, 175193.

Zhang, M., and J. Koplik (1997), Tracer dispersion in three-dimensional multipole flows, Phys. Rev. E, 56, 42444258.

Zheng, C., and G. D. Bennett (2002), Applied Contaminant Transport Modeling, 2nd ed., John Wiley, New York.

Zinn, B., L. C. Meigs, C. F. Harvey, R. Haggerty, W. J. Peplinski, and C. F. Von Schwerin (2004), Experimental visualization of solute transport and mass transfer processes in two-dimensional conductivity fields with connected regions of high conductivity, Environ. Sci. Technol., 38, 3916 3926.

O. A. Cirpka, Swiss Federal Institute of Aquatic Science and Technology, Überlandstr. 133, CH-8600 Dübendorf, Switzerland.

M. Dentz, Department of Geotechnical Engineering and Geosciences, Technical University of Catalonia, E-08034 Barcelona, Spain.

P. A. Kitanidis, Department of Civil and Environmental Engineering, Stanford University, Stanford, CA 94305-4020, USA.

J. Luo, School of Civil and Environmental Engineering, Georgia Institute of Technology, Atlanta, GA 30332-0355, USA. (jianluo@ce.gatech.edu) 